\title{
Mitigating Warranty Risk for Automotive Industry
}

\author{
Ahmed Aljazea*, Ming Luo** and Shaomin $\mathrm{Wu}^{* * *}$ \\ * Kent Business School, Canterbury, Kent CT2 7FS, UK. Email: ama65@kent.uk.ac \\ **Kent Business School, Canterbury, Kent CT2 7FS, UK. Email: m1472@kent.ac.uk \\ ***Kent Business School, Canterbury, Kent CT2 7FS, UK. Email: s.m.wu@kent.ac.uk
}

\begin{abstract}
Warranty is offered by manufacturers as protection and promotional tools. It also gives customers a certain degree of insurance against product failures for a certain period. Although bringing those benefits, it involves various risks originating from various perspectives of the product lifetime cycle. To prepare risk mitigation plans is therefore needed, which is challenging due to the increasing complexity of the product designs and the long warranty period. Accordingly, the decision made to select the mitigation plan involves a high level of uncertainty. This paper develops a plan to mitigate warranty risk based on cumulative prospect theory, which helps warranty decision makers in selecting the optimal mitigation plan.
\end{abstract}

\section{Introduction}

Although the literature of warranty management is vast, it focuses on making financial plans such as estimating the number of the future warranty claims or costs, optimising maintenance policies, among others. In contrast, warranty risk management (WaRM) has received little attention and only been mentioned as an off topic by Díaz \& Márquez (2011), González-Prida \& Márquez (2012) and Costantino et al. (2012).

Mitigating warranty risk as a decision problem is another challenge that has not been investigated in the literature. The risk mitigation process requires decision makers to opt the optimal mitigation plan fulfilled the optimal cost and result based on predefined criteria. In the warranty context, the decision made to select such a plan may involve a high level of uncertainty and risk due to the increasing complexity of the new invitations and long warranty period.

Different multi-criteria decision-making tools (MCDM) such as multi-attribute utility theory (MAUT) and analytic hierarchy process (AHP) can be used to select the optimal warranty risk mitigation plan (Velasquez \& Hester, 2013). However, the existing methods are unable to capture some behavioural aspects of the decision makers towards making the decision under uncertainty and risk, which can influence the final decision.

There are some behavioural decision-making methods such as prospect theory (Kahneman, 1979), regret theory (Bell, 1982), disappointment theory (Bell, 1985), third generation prospect theory (Schmidt et al., 2008) and cumulative prospect theory (Tversky \& Kahneman, 1992) were therefore developed. The cumulative prospect theory (CPT), however, shows its superiority through well describing the decision makers' attitude in four elements: (1) reference dependence; (2) loss aversion; (3) diminishing sensitivity, and (4) probability weighting, and providing the formulas required to calculate values and weights in a clear logic and simple computation process. For this reason, CPT is adapted in this paper to address the decision problem in mitigating warranty risk. Interested readers can refer to (Tversky \& Kahneman, 1992) for more details.

In warranty management practice, some automotive manufacturers may invalidate warranty if the customer fails to comply with the recommended service schedule or if the vehicle continues to be driven when the fault is apparent ${ }^{1}$. It implies that the periodic check-ups are necessary to detect failures at the early stage; after a certain period or after a certain amount of failures reported, WDMs may decide to implement a mitigation plan selected from a pre-specified protocol in response to the occurred warranty claims. The chosen plan at time $t$, however, can affect the future decisions concerning the selection of

\footnotetext{
${ }^{1}$ For example, BMW (2018): https://www.bmw.co.uk/bmw-ownership/servicing-and-repairs/bmwwarranties/new-car-warranty-guidelines
} 
the optimal mitigation plan at the future check-ups. Accordingly, the reference point of the mitigation plans and the WDMs' behaviour may be changed. For example, the loss aversion can be greater at $t_{i+1}$ than $t_{i-1}$ due to some losses experienced in the recent past. This paper models the decision process of risk mitigation with the CPT considering time-varying variables such as the reference point of the costs of warranty mitigation plans and the parameters that capture WDMs' behaviour).

\section{Introduction to CPT, Assumptions and notations}

Cumulative prospect theory (CPT) is a decision making model for descriptive decisions under risk and is introduced by Tversky \& Kahneman (1992).

We make the following assumptions in this paper.

(1) The warranty decision makers (WDM) need to select different mitigation plans to treat warranty related hazards at different time points based on two criteria (a) warranty cost, and (b) manufacturer reputation.

(2) The time to an implement mitigation plan is pre-scheduled.

(3) Three levels of mitigation plans are considered: (a) product recall, (b) selective recall, and (c) corrective rectification.

(4) A mitigation plan can lead to more than one outcome with different probabilities.

\section{Notations:}

$\boldsymbol{O}=\left\{o_{1}\right\}, i=1,2, \ldots m$

$\boldsymbol{P}=\left\{p_{j}\right\}, j=1,2, \ldots, n$

$\boldsymbol{C}=\left\{C_{k}\right\}, k=1,2, \ldots, q$

$\boldsymbol{R}=\left\{R_{i j k}\right\}, i$

$=1,2, \ldots, m ; j$

$=1,2, \ldots, n ; k=1,2, \ldots, q$

$o^{\mathrm{r}}$

$R^{\mathrm{r}}=\left\{R_{k}^{\mathrm{r}}\right\}, k=1,2, \ldots, q$

$\Phi=\left(\mu^{\mathrm{c}}, \mu^{\mathrm{r}}\right)$ vector of mitigation plan costs at different levels.

vector of state probability, where $p_{j}$ denotes the probability of different states of outcomes and $\sum_{j=1}^{n} p_{j}=1$.

criteria that should be considered during the warranty risk analysis, where $C_{k}$ is the $k$ th criterion. The set of $C$ represents the main potential elements that will be influenced once warranty risks have occurred. Two types of criteria are often considered in the decision analysis, costs and benefits (Peng et al., 2011). Thus, we denote $C^{c}$ and $C^{b}$ as the criterion sets of cost and benefit type, respectively, where $C^{c} \cup C^{b}=C$ and $C^{c} \cap C^{b}=\emptyset$. Accordingly, we denote $I^{c}$ and $I^{b}$ as the subscript sets of $C^{c}$ and $C^{b}$ respectively, where $I^{c} \cup I^{b}=\{1,2, \ldots, q\}$ and $I^{c} \cap I^{b}=\emptyset$.

a set of the results of applying a mitigation plan to the impact of a state of the emerged warranty event on $C_{k}$. For example, for warranty cost, a positive value means the expected aggregate warranty cost in the next period is increased.

the reference point of the cost of the mitigation plans. Here, the average warranty cost in the industry can be used as a reference point or the average warranty cost of the product based on the historical record.

vector of the criterion $C_{k}$ reference point, where the average of $C_{k}$ values is deemed as the reference point $R_{k}^{r}$. Two types of criteria are often considered in the decision analysis, costs and benefits (Peng et al., 2011). Thus, we denote $C^{c}$ and $C^{b}$ as the criterion sets of cost and benefit type, respectively, where $C^{c} \cup C^{b}=C$ and $C^{c} \cap C^{b}=\emptyset$. Accordingly, we denote $I^{c}$ and $I^{b}$ as the subscript sets of $C^{c}$ and $C^{b}$ respectively, where $I^{c} U I^{b}=\{1,2, \ldots, q\}$ and $I^{c} \cap I^{b}=\emptyset$.

weight vector of mitigation plan cost and result, where the $\mu^{\mathrm{c}}$ denotes the degree of importance of the spending of different mitigation plans, and the $\mu^{\mathrm{r}}$ denotes the importance degree of the mitigation plan result; $\mu^{\mathrm{c}}+\mu^{\mathrm{r}}=1,0 \leq \mu^{\mathrm{c}}, \mu^{\mathrm{r}} \leq 1$. The value of $\mu^{\mathrm{c}}, \mu^{\mathrm{r}}$ can be assigned by decision makers.

Time of making $i$ th decision.

Expected aggregate warranty cost during $\left[t_{i-1}, t_{i}\right)$. 


\section{Model Development}

The warranty risk mitigation plans will be ranked from the greatest to the smallest, considering $P, O, R$, $o^{\mathrm{r}}(t), R^{\mathrm{r}}$ and $\Phi$. The solution procedure of the warranty decision problem under uncertainty is briefly discussed as the follow:

(1) As the impact on manufacturer reputation criterion is qualitatively determined (ordinal data), we need to transform it into interval data in order to use it in the later steps. Likewise, the impact on warranty cost criterion, it will be transformed into interval data (Guilford, 1975).

(2) The criterion values and mitigation plan costs and their reference points will be normalised through utilising the normalised approach (Kou et al., 2012).

(3) The value function of CPT will be utilised to calculate the value of $R_{i j k}$.

(4) As there are potential interdependences between criteria, i.e. the outcomes of one decision under different criteria are non-additive. Then, the Choquet integral is used to aggregate the outcomes under different criteria of each decision to compare the decisions.

(5) The weighting function of CPT will be used to determine the weight of the objective probability of each potential result obtained from (4).

(6) Based on (4) and (5), the prospect value of each mitigation plan treating a certain state can be calculated.

(7) Based on the prospect values derived from (6), the overall prospect value of each mitigation plan is calculated.

(8) The ranking of warranty risk mitigation plans can be achieved based on the overall prospect value derived from (7).

In the warranty practice, WDMs may schedule several time points to review the warranty claims related issues and respond to the emerged hazards by selecting the optimal mitigation plan, as discussed above. The chosen plan at this time, however, can affect the future decision concerning the determination of the optimal mitigation plan at the future review points. Accordingly, the reference point of the mitigation plans $o^{\mathrm{r}}$ and the parameters values $g_{t_{i}}, l_{t_{i}}$ and $\lambda_{t_{i}}$ may be changed. For example, the loss aversion can be greater at $t_{i+1}$ due to some losses experienced in the recent past.

The criterion values and reference point will be normalised into numbers between 0 and 1 in order to unify them. Let $\overline{o_{l}}$ denote the normalised cost of a warranty mitigation plan. It is then presented as:

where

$$
\bar{o}_{i}=\frac{o^{\max }-o_{i}}{o^{\max }-o^{\min }}, \quad i=1,2, \ldots, m
$$

$$
\begin{aligned}
& o^{\max }=\max \left\{o^{\mathrm{r}}(t), o_{1}, o_{2}, \ldots, o_{m}\right\}, \\
& o^{\min }=\min \left\{o^{\mathrm{r}}(t), o_{1}, o_{2}, \ldots, o_{m}\right\},
\end{aligned}
$$

It can be noticed that $o^{\mathrm{r}}(t)$ is embedded in the Eqs. (2) and (3) in order to maintain the comparability between the reference point of the mitigation plans costs and the $o_{i}$ values. In Eqs. (1)--(3), the costs of mitigation plans are unified into benefit type (Peng et al., 2011; Kou et al., 2012). In other words, the greater $\bar{o}_{i}$ is, the better mitigation plan will be.

Let $\bar{R}_{i j k}$ denote the normalisation of the results $R$ with respect to $C_{k}$. It is calculated as: $\bar{R}_{i j k}=$ $\frac{R_{i j k}-R_{k}^{\min }}{R_{k}^{\max }-R_{k}^{\min }}$, for $i=1,2, \ldots, m ; j=1,2, \ldots, n ; k \in I^{B}$, and $\bar{R}_{i j k}=\frac{R_{k}^{\max }-R_{i j k}}{R_{k}^{\max }-R_{k}^{\min }}$, for $i=1,2, \ldots, m ; j=$ $1,2, \ldots, n ; k \in I^{C}, \quad$ where $\quad R_{k}^{\max }=\max \left\{R_{k}^{r}, R_{11 k}, \ldots, R_{1 n k}, \ldots, R_{m 1 k}, \ldots, R_{m n k}\right\}, \quad R_{k}^{\min }=$ $\min \left\{R_{k}^{r}, R_{11 k}, \ldots, R_{1 n k}, \ldots, R_{m 1 k}, \ldots, R_{m n k}\right\}$, and the reference point $R_{k}^{\mathrm{r}}$ is embedded to maintain the comparison between the $R$ values and their reference points. Similarly, the criterion values are unified into benefit type (Kou et al., 2012). In other words, the greater $\bar{R}_{i j k}$ is the better result $R$ will be. For example, if $R_{i j k}$ denotes the change of warranty cost, a positive value means the cost increased, and a negative value means the cost decreased; then $R_{i j k}$ should be normalized by the cost type, $\bar{R}_{i j k}=$ $\frac{R_{k}^{\max }-R_{i j k}}{R_{k}^{\max }-R_{k}^{\min }}$, as higher $\bar{R}_{i j k}$ can indicate lower cost. If $R_{i j k}$ denotes the change of reputation; then it 
should be normalized by the benefit type, $\bar{R}_{i j k}=\frac{R_{i j k}-R_{k}^{\min }}{R_{k}^{\max }-R_{k}^{\min }}$, as higher $\bar{R}_{i j k}$ can indicate higher reputation. The purpose of nasalization is to measure the criteria in the same scale that higher score indicates a better position the decision located in.

In order to determine the new reference point $o_{t_{i}}$, WDMs may assess the expected value $S_{i}$ of the aggregated warranty cost for the interval $\left[t_{i-1}, t_{i}\right.$ ). This can be computed as (Luo \& Wu, 2018): $E\left[S_{i}\left(t_{i}\right)\right]=N\left(t_{i}\right) Z_{i}$ where $N$ is the number of warranty claims which assumed to follow the nonhomogenous Poission process and $Z$ is the warranty cost.

The reference point of the mitigation plan at the time $t_{i}$ is determined based on the expected aggregate warranty cost $S_{i}$.

$$
o^{\mathrm{r}}(t)=f\left(S_{i}\right)
$$

where $f(0$ can represent the decision maker's attitude on the incurred warranty cost. The function can be monotone increasing, decreasing or other non-monotone forms. Let $\overline{o_{l}^{r}}$ denote the normalised reference point pertaining the mitigation plan cost, it is computed as

$$
\bar{o}^{\mathrm{r}}=\frac{o^{\max }-o^{\mathrm{r}}(t)}{o^{\max }-o^{\min }}
$$

Let $\bar{R}_{k}^{\mathrm{r}}$ denote the normalised reference point of the $C_{k}$ criterion, it is calculated as: $\bar{R}_{k}^{\mathrm{r}}=$ $\frac{R_{k}^{\mathrm{r}}-R_{k}^{\min }}{R_{k}^{\max }-R_{k}^{\min }}$, for $k \in I^{B}$, and $\bar{R}_{k}^{\mathrm{r}}=\frac{R_{k}^{\max }-R_{k}^{\mathrm{r}}}{R_{k}^{\max }-R_{k}^{\min }}$, for $k \in I^{C}$.

Thus, based on the values of $\bar{R}_{i j k}$ and $\bar{R}_{k}^{\mathrm{r}}$, the value of $R$ concerning the criterion $C_{k}$ can be calculated. If $\bar{R}_{i j k} \geq \bar{R}_{k}^{\mathrm{r}}$ then $R$ is deemed as a gain in respect of $C_{k}$ criterion. This gain can be calculated as $\bar{R}_{i j k}-\bar{R}_{k}^{\mathrm{r}}$. In contrast, if $\bar{R}_{i j k}<\bar{R}_{k}^{\mathrm{r}}$ then $R$ is deemed as loss in respect of $C_{k}$ criterion. This loss can be calculated as $\bar{R}_{k}^{\mathrm{r}}-\bar{R}_{i j k}$. In this research, the average of $R_{k}$ is used as a reference point for each criterion. Terzi et al. (2016) found that the population average payoff level is the modal reference point for individuals when they attempt to make a decision under risk.

Let $v_{i j k}$ denote the value of $R$ with respect to $C_{k}$ criterion. Thus, based on the value function of CPT the calculation of $v_{i j k}$ at time $t_{i}$ is computed as:

$$
v_{i j k}(t)=\left\{\begin{array}{c}
\left(\bar{R}_{i j k}-\bar{R}_{k}^{r}\right)^{g_{k}(t)}, \quad \bar{R}_{i j k} \geq \bar{R}_{k}^{\mathrm{r}} \\
-\lambda_{k}(t)\left(\bar{R}_{k}^{r}-\bar{R}_{i j k}\right)^{l_{k}(t)}, \bar{R}_{i j k}<\bar{R}_{k}^{\mathrm{r}}
\end{array}, i=1,2, \ldots, m ; j=1,2, \ldots, n ; k=1,2, \ldots, q\right.
$$

where $g_{k}(t)$ and $l_{k}(t)$ are time-varying parameters which represent the time-varying levels of riskaversion of WDMs over the gain and risk-seeking over the loss with respect to $C_{k}$ criterion, respectively. The $\lambda_{k}(t)$ is a time-varying parameter representing the loos-aversion attitude of WDMs with respect to $C_{k}$ criterion, where $0<g_{k}(t), l_{k}(t) \leq 1, \lambda_{k}(t)>1, k=1,2, \ldots, q$. Such parameters can be determined through experiments.

The $R$ can be then assessed based on the values resulting from Eq. (9). Let $v_{i j}$ denote the value of the result $R_{i j}$, then it can be assessed based on the aggregation of $v_{i j 1}, v_{i j 2}, \ldots, v_{i j q}$. However, we assume there is an interdependence between criteria, e.g., between warranty cost criterion and manufacturer reputation criterion. Therefore, the Choquet integration model is used to aggregate $v_{i j 1}, v_{i j 2}, \ldots, v_{i j q}$ into the value of $R_{i j}$. Accordingly, these values are ranked in a decreasing order through a comparison between them. As a result, the ranking order is presented as $v_{i j(1)} \leq v_{i j(2)} \leq$ $\cdots \leq v_{i j(q)}$, where $v_{i j(k)}$ denote the $k$ th smallest result among $v_{i j 1}, v_{i j 2}, \ldots, v_{i j q}, k=1,2, \ldots, n$. Let $C_{i j(k)}$ denote the criterion corresponding to $v_{i j(k)}$, where $C_{i j(k)} \in C$. Let $u\left(E_{i j(k)}\right)$ denote the importance degree of criterion subset $E_{i j(k)}=\left\{C_{i j(k)}, C_{i j(k+1)}, \ldots, C_{i j(q)}\right\}$. The importance can be assigned directly by decision makers or indirectly by using some existing methods such as analytic hierarchy process method (AHP) (Saaty, 2008). It is clear that $E_{i j(1)}=\left\{C_{1}, C_{2}, \ldots, C_{q}\right\}=\Omega$ and $E_{i j(q+1)}=\emptyset$. Hence, the value of $u\left(E_{i j(1)}\right)=1$ and $u\left(E_{i j(q+1)}\right)=0$. Based on that, the potential value of $v_{i j}(t)$ at time $t_{i}$ can be calculated as: 


$$
v_{i j}(t)=\sum_{k=1}^{q} v_{i j, k}\left[u\left(E_{i j, k}\right)-u\left(E_{i j, k+1}\right)\right], \quad i=1,2, \ldots, m ; j=1,2, \ldots, n
$$

The prospect value of each mitigation plan can be calculated based on the values of $v_{i 1}, v_{i 2}, \ldots, v_{i n}$ obtained from Eq. (10). To do so, these values are ranked in an increasing order of $v_{i j}$ which is determined through comparing $v_{i 1}, v_{i 2}, \ldots, v_{i n}$. Hence, the ranking result should be presented as $v_{i(1)} \geq$ $v_{i(2)} \geq \cdots \geq v_{i(n)}$ or $\left(v_{i(n)} \geq v_{i(n-1)} \geq \cdots \geq 0 \geq \cdots \geq v_{i(1-m)}, v_{i(-m)}\right)$ where $v_{i(k)}$ is the $k$ th greatest among $v_{i(1)}, v_{i(2)}, \ldots, v_{i(n)}, k=1,2, \ldots, n$. Correspondingly, the probabilities of the states are re-indexed as $p_{i(1)}, p_{i(2)}, \ldots, p_{i(n)}, p_{i(k)} \in P, k=1,2, \ldots, n$. The prospect value $V_{i}$ of each mitigation plan at time $t_{i}$ is then computed as:

$$
V_{i}(t)=\sum_{k=0}^{n} v_{i, k} \pi_{i, k}^{+}+\sum_{k=-m}^{0} v_{i, k} \pi_{i, k}^{-}, \quad i=1,2, \ldots, m,
$$

Denote weighting function as $(x, \mu)=x^{\mu} /\left(x^{\mu}+(1-x)^{\mu}\right)^{1 / \mu}$, and hence the decision weights $\pi_{i, k}^{+}$and $\pi_{i, k}^{+}$for gains and losses, respectively, can be computed as the following (Tversky \& Kahneman, 1992):

$$
\begin{array}{cc}
\pi_{i, k}^{+}=w\left(\sum_{z=k}^{n} p_{i, z}, \mu\right)-w\left(\sum_{z=k+1}^{n} p_{i, z}, \mu\right), & 0 \leq k \leq n-1 \\
\pi_{i, k}^{-}=w\left(\sum_{z=-m}^{k} p_{i, z}, \mu\right)-w\left(\sum_{z=-m}^{k-1} p_{i, z}, \mu\right), & 1-m \leq k<0
\end{array}
$$

where $x$ is the objective probabilities and $0.27<\mu<1$, and $w(\cdot)$ is monotonic depicting the inverse S-shapes (Tversky \& Kahneman, 1992). These parameter $\mu$ reflects the behaviour of decision makers such as overweighting the outcomes with low probabilities and underweighting the outcomes with moderate and high probabilities, and it is determined through experiments (Tversky \& Kahneman, 1992).

Now, we need to calculate the cost of each mitigation plan to assess the overall prospect value $O V_{i}$. To do so, we need $\bar{o}_{l}$ and $\bar{o}^{\text {r }}$. It is important to emphasise that these costs are transformed into benefit type based on the Eqs. (1) and (7). If the $\overline{o_{l}} \geq \bar{o}^{r}$, then there is $\overline{o_{l}} \leq \bar{o}^{r}$ and hence the mitigation plan can be considered as a gain computing as $\overline{o_{l}}-\bar{o}^{r}$, while if $\bar{o}_{l}<\bar{o}^{r}$, then there is $\bar{o}_{l}>\bar{o}^{r}$ which can be deemed as a loss calculating as $\bar{o}^{r}-\bar{o}_{l}$. Accordingly, let $V_{i}^{\mathrm{c}}$ denote the value cost of a mitigation plan, according to Eq.(2) the $V_{i}^{\mathrm{c}}$ can be computed as:

$$
V_{i}^{\mathrm{c}}=\left\{\begin{array}{rl}
\left(\overline{o_{l}}-\bar{o}^{\mathrm{r}}\right)^{g^{\mathrm{c}}}, & \overline{o_{l}} \geq \bar{o}^{\mathrm{r}} \\
-\lambda^{\mathrm{c}}\left(\bar{o}^{\mathrm{r}}-\bar{o}_{l}\right)^{l^{\mathrm{c}}}, & \overline{o_{l}}<\bar{o}^{\mathrm{r}}
\end{array} \quad i=1,2, \ldots, m,\right.
$$

As the cost of each mitigation plan is certain, then the value of cost can be treated as the prospect value of cost. Therefore, the overall prospect value $O V_{i}$ of each mitigation plan at time $t_{i}$ can be computed by:

$$
O V_{i}(t)=\mu^{\mathrm{r}} V_{i}+\mu^{\mathrm{c}} V_{i}^{\mathrm{c}} \quad, i=1,2, \ldots, m
$$

Based on the result of $O V_{i}$, the greater overall prospect value is the better mitigation plan will be. Accordingly, the mitigation plans will be ranked according to their overall prospect value and selected the greatest one to mitigate the warranty risks.

Then, the integrated mitigation policy including the mitigation plans selected at each time can be determined. The overall value of an integrated mitigation policy is $\boldsymbol{O V}=\sum_{i}^{n} O V_{i}$.

the optimal integrated mitigation policy can be determined in further research. The time-varying property of this model is represented by the change in the reference point of the cost of the mitigation plans $o_{i}^{r}$ and the time-varying parameters related the decision maker's behaviour under risk and uncertainty. In further research, for the optimization problem, the objective function is $\boldsymbol{O V}=\sum_{i}^{n} O V_{i}$, which should be maximized with the constraint is $\sum_{i=1}^{k} o_{i} \leq \boldsymbol{\gamma}$, where $\gamma$ is a prespecified total budget. 


\section{Conclusion}

Although warranty plays a significant role nowadays, it may also cause substantial financial and reputational losses for manufacturers. As such, a better planning for warranty risks is extremely important. WDMs therefore should consider proactive plans for mitigating warranty risks when they have occurred.

As such, this paper focuses mainly on developing a tool aiding WDMs to select the suitable mitigation plan based on different criteria. The following points, therefore, have been achieved:

1) The average warranty cost in the industry is used to be the reference point for the mitigation plan costs.

2) Since the reference point of mitigation plans costs and the estimated parameters capturing the WDMs behaviour can change over the warranty period, the time dependence is integrated to the CPT-WaRM to respond to such changes.

This model may aid WDMs in evaluating and ranking different mitigation plans when considering those psychological aspects that may affect the final decision such as reference dependence, loss aversion, diminishing sensitivity, and probability weighting. In addition, the optimal mitigation plan can be determined at different revision points of warranty claims based on the first selected plan.

\section{References}

Bell, D. E. 1982. Regret in decision making under uncertainty. Operations Research, 30, 961-981.

Bell, D. E. 1985. Disappointment in decision making under uncertainty. Operations Research, 33, 127.

Costantino, F., De Minicis, M., González-Prida, V. \& Crespo, A. 2012. On the use of Quality Function Deployment (QFD) for the identification of risks associated to warranty programs. ESREL Conference.

Díaz, V. G. \& Márquez, A. C. 2011. On the risks and costs methodologies applied for the improvement of the warranty management. Journal of Service Science and Management, 4, 191.

González-Prida, V. \& Márquez, A. C. 2012. A framework for warranty management in industrial assets. Computers in Industry, 63, 960-971.

Guilford, J. P. 1975. Psychometric methods, Bombay, Tata McGraw-Hill.

Kahneman, D. 1979. Prospect theory: An analysis of decisions under risk. Econometrica, 47, 278.

Kou, G., Lu, Y., Peng, Y. \& Shi, Y. 2012. Evaluation of classification algorithms using MCDM and rank correlation. International Journal of Information Technology \& Decision Making, 11, 197225.

Luo, M. \& Wu, S. 2018. A mean-variance optimisation approach to collectively pricing warranty policies. International Journal of Production Economics, 196, 101-112.

Peng, Y., Kou, G., Wang, G. \& Shi, Y. 2011. FAMCDM: A fusion approach of MCDM methods to rank multiclass classification algorithms. Omega, 39, 677-689.

Saaty, T. L. 2008. Decision making with the analytic hierarchy process. International journal of Services Sciences, 1, 83-98.

Schmidt, U., Starmer, C. \& Sugden, R. 2008. Third-generation prospect theory. Journal of Risk and Uncertainty, 36, 203.

Terzi, A., Koedijk, K., Noussair, C. N. \& Pownall, R. 2016. Reference point heterogeneity. Frontiers in Psychology, 7, 1347.

Tversky, A. \& Kahneman, D. 1992. Advances in prospect theory: Cumulative representation of uncertainty. Journal of Risk and Uncertainty, 5, 297-323.

Velasquez, M. \& Hester, P. T. 2013. An analysis of multi-criteria decision making methods. International Journal of Operations Research, 10, 56-66. 